\title{
Collaborative Dispatching of Commercial Vehicles
}

\author{
Asvin Goel and Volker Gruhn \\ Chair of Applied Telematics and e-Business, Computer Science Faculty \\ University of Leipzig \\ Klostergasse 3, 04109 Leipzig, Germany \\ \{goel,gruhn\}@ebus.informatik.uni-leipzig.de
}

\begin{abstract}
Collaborative dispatching allows several dispatchers to view the routing solution as a dynamic model where changes to the vehicle routes can be made in real-time. In this paper we discuss implications of collaborative dispatching on real-time decision support tools for motor carriers. We present a collaborative dispatching system which uses real-time information obtained from a telematics system. Messages sent from the vehicles are automatically analysed and actual data, such as exact arrival and departure times, as well as discrepancies between actual and planned data are identified. The collaborative dispatching system not only allows several dispatchers to concurrently modify the schedule, but also a dynamic optimisation method. The optimisation method is capable of taking into account that input data may change at any time and that dispatchers can concurrently modify the schedule and may add or relax certain constraints relevant to the optimisation model.
\end{abstract}

Keywords-Vehicle Routing, Decision Support Systems, Intelligent Transportation Systems

\section{INTRODUCTION}

Due to globalisation, liberalisation of markets, deregulation in the transport sector, and the increasing commitment to the just-in-time philosophy, competition between motor carriers and expectations on punctuality, reliability, flexibility, transparency and quality of transportation services have increased significantly and will increase even more in the future. In most real-life applications changes in data concerning the transportation systems can occur at any time. Examples for such dynamic changes in the problem data are changing traffic conditions and delays due to congestion. These changes can be transmitted to the dispatching office by the vehicles if they are equipped with telematics systems, typically consisting of input devices and a positioning system. Other dynamic data may be the arrival of new transportation requests which have to be assigned to vehicles considering the current state of the transportation system. In order to successfully face today's challenges it is essential that motor carriers use real-time computer-based decision support systems.

As humans can only process a limited amount of information efficiently motor carriers typically deal with the challenge of juggling all this information by dividing the covered area into regions of manageable size. As a result, decisions made by a dispatcher responsible for one region may not take into account transportation requests and resources on the other side of the regional boundary. Obviously, there is a potential to improve planning decisions concerned with transportation requests and resources in proximity of the regional boundary.
The high volume of information that needs to be handled when regional boundaries are avoided seems to be an obvious application for computerised decision support as computers are very good at processing vast quantities of information. Humans, on the other hand, are very good at challenging the information that is in the computer and augmenting computerprovided solutions with head knowledge.

According to [1] collaborative dispatching allows several dispatchers to view the routing solution as a dynamic model where changes to the vehicle routes can be made in real-time. In this paper a collaborative dispatching system is presented which has the following features: a) it is not necessary to divide the covered area into multiple regions, b) real-time information transmitted by telematics systems is automatically analysed and used to identify disturbances in the transportation processes, c) a dynamic planning system is used to support the dispatchers by continuously calculating optimised schedules.

The remainder of this paper is organised as follows. First, we give a short overview over related work. Then, we discuss issues related to collaborative dispatching. We present the architecture of an collaborative dispatching system and the functions of its subsystems. Eventually, we present some practical experiences.

\section{RELATED WORK}

Recent trends in the logistics industry, such as the increasing commitment to the just-in-time philosophy and the increasing use of information and communication technologies as well as the impact of these trends on the trucking industry have been discussed by [2]. According to [2] positioning of vehicles, electronic data interchange (EDI) and the internet can provide the necessary information required to achieve real-time computer-based decision support.

Today, only a few decision support tools can be found that integrate real-time information. Many of the systems available today still require that the data is manually transfered between the logistics application and a telematics system which provides real-time information from the vehicles - a time consuming and error prone task. Yet, some techniques to use real-time information to support the management of commercial vehicle operations have been developed. For example, [3] propose a concept for managing a truck fleet through cell-phones and the internet. [4] have proposed a mobile communication system, which focuses on the driver/dispatcher interaction and the integration with logistics software. [5] present a concept for 
the integration of an off-the-shelf telematics systems into the carrier's IT infrastructure. The presented system automatically analyses messages sent by the vehicles. The analysed information is then automatically made available to the logistics system.

Computer-based planning tools for motor carriers have been surveyed by [1]. One finding is that collaborative dispatching is still an area in which further research must be conducted. Collaborative dispatching allows several dispatchers to view the routing solution as a dynamic model where changes to the vehicle routes can be made in real-time. This assumes that some wireless communication technique can be used to inform drivers whenever new tasks are assigned to them. Collaborative dispatching achieves its power by specialised actors who cooperatively use different problem knowledge and solution methods. Besides of the human dispatchers another specialised actor can be incorporated into the collaborative dispatching system: the computer with its advantage in processing vast quantities of information. The interaction between human dispatcher and computer-based planning has been studied by [6] who presented a concept for interactive dispatching. This framework, however, does not consider several dispatchers who may concurrently modify the schedule and assumes a "step-by-step" approach in which the human dispatcher and the computer-based problem solver alternately change the schedule.

According to [7] there is usually a big gap between information available to the dispatchers who speak directly to drivers and shippers and the information available to the computer. Although, real-time information systems can improve the availability of information it is unlikely that all relevant information can become available to the computer as a result of the sheer cost of getting information into the computer. Therefore, motor carriers generally rely on human dispatchers to make the appropriate decisions in real-time. The impact of user noncompliance, which occurs when users do not adopt all of the recommendations of an optimisation model has been studied by [8].

The development of dynamic optimisation methods is an active research field and several algorithms have been proposed recently, for example, [9] and [10]. These methods are event-driven and modify solution only when new orders arrive or a transportation process has been completed. Iterative improvement procedures for continuous dynamic planning are presented by [11] and [12]. These algorithms are characterised by the capability of handling a variety of practical requirements of real-life problems and fast response times. They can be used in collaborative dispatching systems where several dispatchers and a dynamic planning system modify schedules concurrently.

\section{Collaborative Dispatching}

Collaborative dispatching requires that several actors, human dispatchers or a dynamic planning system, may concurrently try to modify the same part of a solution. Therefore, it is essential to have efficient methods which maintain data consistency, as otherwise e.g. a transportation request may be assigned to two different vehicles by two actors. Pessimistic locking can be used to prevent concurrent modifications concerning the same transportation request or tour. In pessimistic locking, as described in [13] and [14], a data record to be updated is locked in advance. Once an actor has locked a data record he can make the required changes, and then commit or rollback. During the commit or rollback the lock is automatically dropped. Other actors who want to acquire a lock of the same data record during this process will have to wait until the current lock is dropped. The pessimistic locking scheme, although very simple and safe, does not fit well for collaborative dispatching. A dynamic planning system may continuously try to calculate optimised schedules, and thus, would have to lock all data records during optimisation. This would prevent the dispatchers from performing any manual changes to the schedule.

Optimistic locking, as described in [13] and [14], is more convenient for collaborative dispatching. Optimistic locking does not lock data records when they are read, and proceeds on the assumption that the data records being updated will not be changed. To ensure data consistency the optimistic locking scheme we propose involves reading a Transaction Control Number (TCN) along with each data record representing an order or a tour. When the schedule is changed by the dispatchers or the dynamic planning system, the TCNs of the respective order and the tour are written back to the database when the record is updated. A pre-update trigger checks the value of the updated TCNs against those held in the database. If the TCNs do not match the transaction is rejected. If the TCNs match no other transactions have updated the data records since they were read. The TCNs of orders and tours are incremented with each successful update. The optimistic locking scheme allows several actors to concurrently modify the schedule and it prevents lockouts which may occur in pessimistic locking when one dispatcher selects a data record for update, and then leaves for lunch without finishing or aborting the transaction. In a lockout all other actors who may want to modify that data record are forced to wait until the transaction is completed, or until the data base administrator kills the offending transaction and releases the lock.

Dynamic planning requires a precise representation of the real-life problem. However, discrepancies between model representation and real-life problem arise as a result of the sheer cost of getting information into the computer [7]. Telematics systems can be used to improve the timely availability of information regarding the actual transportation processes and electronic data interchange (EDI) can be used to integrate information systems of the shippers, e.g. for obtaining all relevant data regarding transportation requests and customer locations. Despite of the improved possibilities of getting the data into the model, the information is generally not only incomplete but also imprecise. A shipper, for example, may ask that a load be picked up in the morning before noon, when his dock is not as busy. In the model such restrictions are modelled as time window constraints. The computer system has no way of interpreting whether such a request for early 

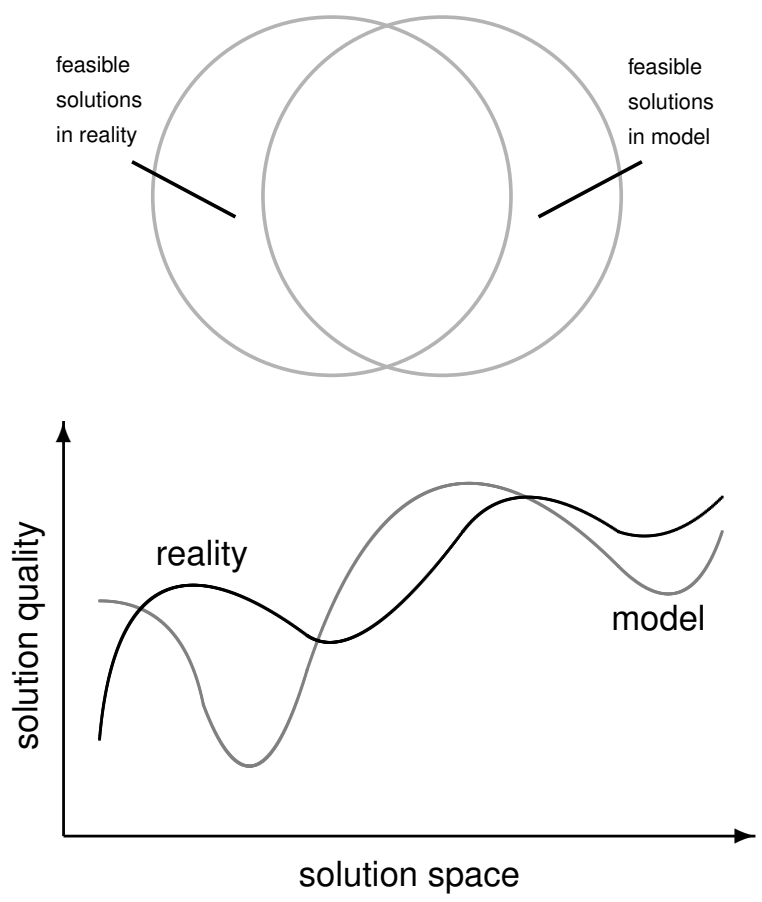

Fig. 1. Differences between real-life problem and model

pickup is a hard constraint or whether the shipper was only trying to express a preference.

As illustrated in figure 1 the impreciseness of the model representation results in two fundamental problems:

- some solutions which are feasible according to the model may not be feasible in reality and vice versa,

- a solution with high quality in the model may not have the same high quality in reality.

Due to the impreciseness of any model representation of the real-life problem and the fact that a significant amount of relevant information is not available to the computer, but only to the dispatchers who are in direct contact with drivers and shippers, model recommendations cannot always be fully applied in collaborative dispatching. According to [8] several motor carriers report that the average usage of model recommendations is below $60 \%$, and good performance is considered around $70 \%$. A collaborative dispatching system must be capable of considering that some schedules may be feasible according to the analytical model but infeasible in the real-life problem and vice versa. According to [6] this can be achieved by allowing dispatchers to modify the analytical model by adding constraints to the problem in a way that those real-life requirements which are violated can be considered in the analytical model. In addition selected constraints can be removed if necessary. Furthermore, dispatchers may want to fix parts of the schedule such that they are not modified by the dynamic planning system or other dispatchers. In particular, if a task has been permanently assigned to a driver and the driver has already been informed about this task the corresponding order must not be assigned to another vehicle.

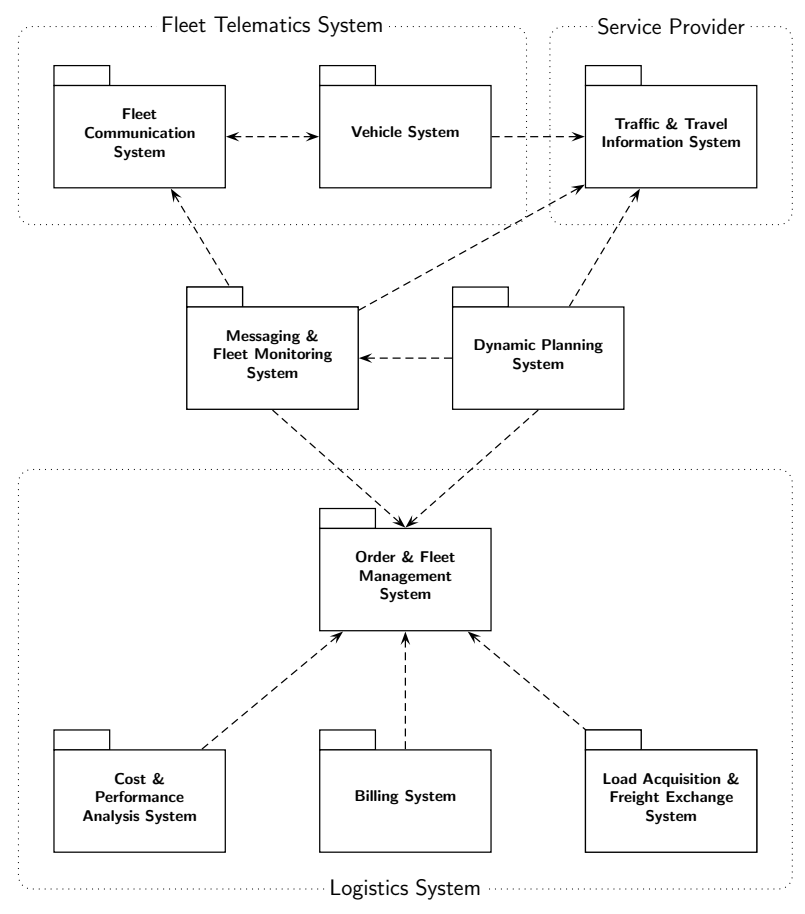

Fig. 2. System architecture

\section{SYSTEM ARCHITECTURE}

An IT-infrastructure for real-time decision support is presented by [5] and illustrated in figure 2 using the Unified Modeling Language. The core of the system is the Order $\&$ Fleet Management System (OFMS) which has the central role concerning the management of orders and vehicles. A Messaging \& Fleet Telematics System (MFMS) is connected to the OFMS and a Fleet Telematics System consisting of mobile Vehicle Systems (VS) and a stationary Fleet Communication System (FCS). The MFMS exchanges information between the FCS and the OFMS analyses incoming messages and puts the resulting data in context to the data stored in the OFMS. A Dynamic Planning System (DPS) can use the hereby obtained real-time information to optimise the schedule considering actual data concerning the vehicle fleet and orders. The MFMS, DPS, and VS may be connected to a Traffic \& Travel Information System in order to consider actual traffic conditions. Several other subsystems can be connected to the OFMS, for example, a Billing System for the generation of invoices, a Cost \& Performance Analysis System for the identification of unnecessary costs and performance measurements of the carrier's operations, and a Load Acquisition \& Freight Exchange System for the acquisition of additional load or the employment of external carriers for loads which cannot be efficiently served by self-operated vehicles. These subsystems, however, are not in the scope of this work.

The OFMS provides all necessary functionalities for the dispatchers to manage the carrier's operations. Dispatchers can store and retrieve all necessary data concerning vehicles and orders as well as the current schedule. The OFMS supports 
the dispatchers by providing graphical user interfaces where filtered information necessary for decision making are presented. The software architecture of the OFMS follows the client/server paradigm [15] and the optimistic locking scheme described above allows several dispatchers to concurrently modify the schedules without the need to divide the covered area into multiple regions. The MFMS and the DPS take over the role of the dispatchers and act as an additional client of the OFMS server. Whenever the MFMS identifies any discrepancies between planned and actual data it adjusts the data in the OFMS. This allows the dispatchers to concentrate on the information received from the vehicles which cannot be unambiguously interpreted by the MFMS. The DPS continuously calculates improved schedules considering the actual conditions which have been entered into the OFMS by the dispatchers and the MFMS as well as manual decisions concerning the planned schedule. In the next sections the MFMS and the DPS are described in more detail.

\section{The Messaging \& Fleet Monitoring System}

The Messaging \& Fleet Monitoring System uses the planned data in the OFMS to instruct drivers about their new tasks. Furthermore, it uses the actual data transmitted by the vehicles in order to revise the planned data in the OFMS and thus, provides all relevant data for real-time dispatching. In order to do so it connects to the FCS and analyses all incoming messages according to their potential content. The information obtained is compared with the planned data in the OFMS. If any discrepancies or unexpected incidents are identified the planned data is revised by the MFMS and dispatchers are automatically notified. Furthermore, the MFMS detects the arrival and departure times at handling locations, calculates expected arrival times considering the current vehicle position. Dispatchers can use the revised data to adjust the schedules and to initiate countermeasures if they identify any disturbances in the transportation processes. A more detailed description of the MFMS can be found in [5] and is not in scope of this paper.

\section{The Dynamic Planning System}

With the availability of reliable information obtained by the MFMS a Dynamic Planning System (DPS) can be used to calculate new tours dynamically as illustrated in figure 3. First, the DPS gets a snapshot of the current schedule. This snapshot may be infeasible according to the analytical model and the real-life problem. This may occur due to disturbances of transportation processes identified by the MFMS, e.g. caused by a vehicle break-down or a significant delay. In some cases infeasibilities can be automatically resolved. For example, if a delay due to congestion is detected the revised arrival times at pickup or delivery locations may be infeasible. If the assignment of the respective order to the tour is not fixed by the dispatchers the order can be removed from the tour. Obviously, all orders which already have been partially served must not be removed and are automatically fixed to the tour. If all orders with infeasible arrival times are removed

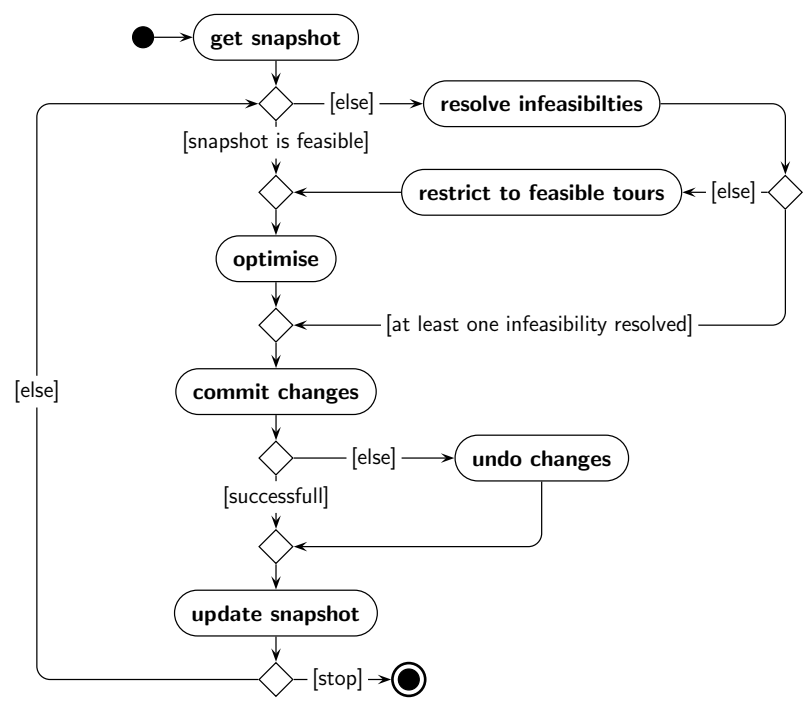

Fig. 3. Dynamic planning

the remaining tour is feasible and can be considered by the optimisation method. Any changes to the schedule have to be committed before the optimisation method is invoked. If no infeasibility can be automatically resolved, all infeasible tours and the orders assigned to these tours are removed from the optimisation model as they can not be properly handled by the optimisation method. Thus, when the optimisation method is invoked only feasible tours, orders assigned to feasible tours, and unscheduled orders are considered.

Using the optimistic locking scheme, the DPS tries to commit an optimised solution or any other changes made to the schedule. If successful the current snapshot is updated in order to consider all changes in the data made by the dispatchers or the MFMS. Otherwise, the changes made on the snapshot by the DPS are undone before the snapshot is updated. The DPS continues with the next iteration until some stopping condition is met - which of course never is the case if the system is used in a rolling planning horizon.

The dynamic optimisation of schedules is a nontrivial task. First, the underlying optimisation model must be capable of handling the real-life requirements. Most optimisation models in literature, however, oversimplify the problems that occur in practise, as pointed out by [16] fifteen years ago. Although real-life problems are receiving increasing attention this is still valid today, as stated by [17] "More effort has gone into methods for reducing the cost of solutions than supporting rich models. However, the problems faced in industry often require rich models ...". Second, many optimisation methods need a lot of time to calculate improving solutions - even for the simple models. As dispatchers may concurrently modify the schedule or instruct drivers about new tasks and as the MFMS may detect changed actual data with respect to the tours and orders considered in the optimisation model, the DPS must have fast response times. That is, the time needed by the DPS to optimise the schedule must be very fast. Otherwise, it would 
be very unlikely that the optimised schedule can be committed successfully.

Large Neighbourhood Search (LNS), presented by [18] has proven to be well suited for rich vehicle routing problems. The basic idea of LNS is to start with an initial solution and to remove $k$ orders from their tours. The number $k$ of orders to be removed can be varied to increase diversification of the search. After these $k$ orders are removed an insertion method tries to re-insert unscheduled orders. In other words, a part of the current schedule remains unchanged and the other part is reoptimised. Although the algorithm proposed by [18] is not very fast a similar approach can be used within the collaborative dispatching system. To ensure fast response times [11] and [12] propose to use fast insertion methods to be used within the LNS iterations. The algorithms proposed are characterised by response times of less than one second even for problems with hundreds of vehicles and several hundreds of orders. They are capable of handling various complexities arising in real-life problems, for example time window restrictions, a heterogeneous vehicle fleet with different travel times, travel costs and capacity. The methods presented in [12] can also take into account restrictions to drivers' working hours as regulated by EU social legislation. The requirements of collaborative dispatching that dispatchers may partially fix solutions can also be handled by the algorithms. A tour or an unscheduled order which is fixed by the dispatchers can simply be removed from the optimisation model. A scheduled order which is fixed to the tour of a vehicle must not be removed from the tour by the DPS, although the DPS may modify other parts of the tour. This can be enforced within the LNS method by preventing the fixed order to be removed from the tour and by using modified time window constraints such that the service times are not modified if another order is inserted to the tour.

\section{PRACTICAL EXPERIENCES}

Georgi Transporte $\mathrm{GmbH}$ is a German motor carrier specialised in the road transport of so-called air-cargo between European airports. The carrier operates 140 vehicles equipped with mobile fleetec III systems [19] which communicate with the stationary DATAfleet system [19]. In a first step we implemented the optimistic locking scheme into the existing OFMS to allow all dispatchers to modify any tour and any order concurrently. The MFMS was realised in order to provide the OFMS with up-to-date information about all transportation processes. After the MFMS was running for one year we asked Georgi Transporte GmbH about their experiences. They reported that the dispatchers were significantly relieved and at the same time the information flow was significantly improved. The improved availability of information eased getting a better overlook over the actual situation and thus, dispatchers were supported in managing the carrier's operations. Unfortunately, no information about a possible reduction of empty mileage and costs resulting from better decisions accompanied with the improved information supply was given to us.

With the MFMS the prerequisites for dynamic planning were provided. The Dynamic Planning System, however, was

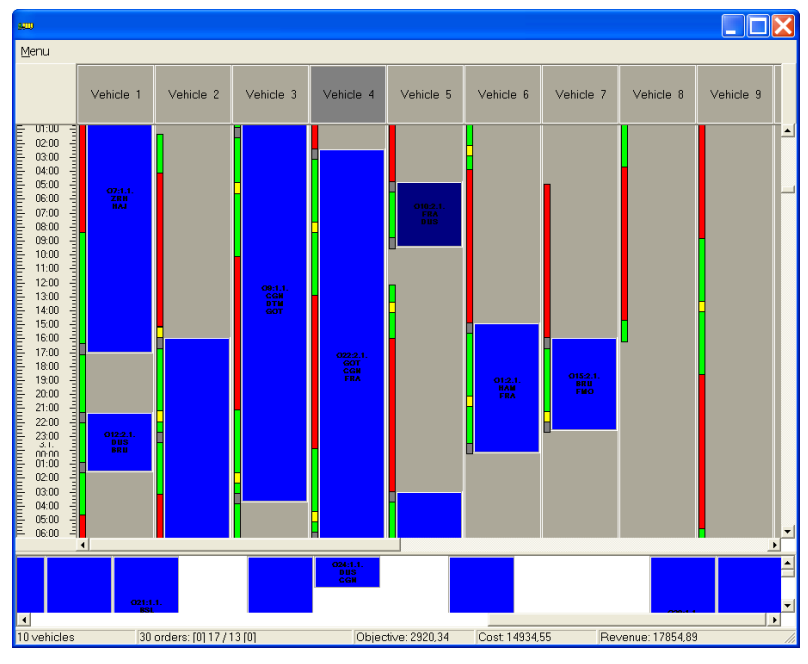

Fig. 4. Prototype of the Dynamic Planning System

only developed as a prototype and is not yet in operation. The prototype of the DPS allows interactive optimisation, that is, it allows the dispatchers to concurrently fix parts of the solution and to manually assign orders to tours or to remove orders from tours.

Figure 4 shows a screenshot of the prototype giving an overview of the current schedule and the drivers' working hours including driving times and daily rest periods. The tour of Vehicle 4 and the order assigned to Vehicle 5 are manually fixed by the dispatchers and will not be changed by the DPS. The DPS optimises the schedule considering all changes made by the dispatchers and new orders entered into the system. Computational experiments have shown that the LNS algorithms presented by [11] and [12], have response times of less than one second for problems with hundreds of vehicles and several hundreds of orders. The solution quality compared to event driven methods, which simply insert new orders when the become known, is significantly higher.

\section{CONCLUSIONS}

In this paper we have presented a collaborative dispatching system for motor carriers. The system uses real-time information and is connected to a telematics systems. Messages sent from the vehicles are automatically analysed and actual data, such as exact arrival and departure times, as well as discrepancies between actual and planned data are identified. Thus, up-to-date information required for dynamic planning is provided. In the collaborative dispatching system it is not necessary to divide the covered area into multiple regions. The optimistic locking scheme allows dispatchers and the Dynamic Planning System to concurrently modify schedules. Dispatchers can furthermore add or relax constraints with respect to the optimisation model, and to modify input data as changes become known. Our practical experiences have shown that the Messaging \& Fleet Monitoring System has significantly improved the availability of up-to-date information. We have developed a prototype of the Dynamic Planning 
System presented in this paper which can be integrated into the carrier's IT system. It will be interesting to see and evaluate the Dynamic Planning System when it is used in practise. Computational experiments have shown that, although response times were less than one second, schedules can be improved significantly.

\section{REFERENCES}

[1] E. Baker, "Evolution of microcomputer-based vehicle routing software," in The Vehicle Routing Problem, P. Toth and D. Vigo, Eds. SIAM Monographs on Discrete Mathematics and Applications, Philadelphia, 2002, pp. 353-361.

[2] J. Roy, "Recent trends in logistics and the need for real-time decision tools in the trucking industry," in HICSS '01: Proceedings of the 34th Annual Hawaii International Conference on System Sciences (HICSS34)-Volume 3. IEEE Computer Society, 2001, p. 3031.

[3] E. Erkens and H. Kopfer, "WAP-LOG: Ein System zur mobilen Fahrzeugeinsatzsteuerung und Auftragsfortschrittkontrolle," In: Logistik Management - Supply Chain Management und e-Business, Teubner Stuttgart, pp. 293-304, 2001.

[4] V. Gruhn, R. Ijioui, M. Hülder, and L. Schöpe, "Mobile communication systems for truckage companies," in The Second International Conference on Mobile Business, G. Giaglis, Ed. Austrian Computer Society, 2003, pp. 337-344.

[5] A. Goel and V. Gruhn, "Integration of telematics for efficient management of carrier operations," in Proceedings of the IEEE International Conference on e-Business Engineering (ICEBE 2005), 2005, pp. 404408.

[6] H. Kopfer and J. Schnberger, "Interactive solving of vehicle routing and scheduling problems: Basic concepts and qualification of tabu search approaches," in HICSS '02: Proceedings of the 35th Annual Hawaii International Conference on System Sciences (HICSS-35)-Volume 3. IEEE Computer Society, 2002, p. 84.
[7] W. Powell, A. Marar, J. Gelfand, and S. Bowers, "Implementing realtime optimization models: A case application from the motor carrier industry," Operations Research, vol. 50, no. 4, pp. 571-581, 2002.

[8] W. Powell, M. Towns, and A. Marar, "On the value of optimal myopic solutions for dynamic routing and scheduling problems in the presence of user noncompliance," Transportation Science, vol. 34, no. 1, pp. 67$85,2000$.

[9] J. Yang, P. Jaillet, and H. Mahmassani, "Real-time multi-vehicle truckload pickup-and-delivery problems," Transportation Science, vol. 38, no. 2, pp. 135-148, 2004.

[10] B. Fleischmann, S. Gnutzmann, and E. Sandvo, "Dynamic vehicle routing based on on-line traffic information," Transportation Science, vol. 38 , no. 4, pp. 420-433, 2004

[11] A. Goel and V. Gruhn, "Large Neighborhood Search for rich VRP with multiple pickup and delivery locations," in Proceedings of the 18th Mini EURO Conference on VNS (MEC-VNS). ISBN 84-689-5679-1, 2005.

[12] — "Solving a dynamic real-life vehicle routing problem," in Proceedings of the International Scientific Annual Conference on Operations Research (OR2005). Springer (to appear), 2005, pp. 369-374.

[13] R. Elmasri and S. Navathe, "Fundamentals of database systems," Third Edition, Addison-Wesley, Reading, Massachusetts, 2000.

[14] G. Thornton, "Optimistic locking with concurrency in oracle," http://www.orafaq.org/papers/locking.pdf [last accessed 03.09.2005], 2001.

[15] S. Lewandowski, "Frameworks for component-based client/server computing." ACM Computing Surveys, vol. 30, no. 1, pp. 3-27, 1998.

[16] L. Bodin, "Twenty years of routing and scheduling," Operations Research, vol. 38, no. 4, pp. 571-579, 1990

[17] P. Kilby, P. Prosser, and P. Shaw, "A comparison of traditional and constraint-based heuristic methods on vehicle routing problems with side constraints," Constraints, vol. 5, pp. 389-414, 2000.

[18] P. Shaw, "A new local search algorithm providing high quality solutions to vehicle routing problems," Technical report, APES group, Department of Computer Sciences, University of Strathclyde, Glasgow, Scottland, 1997.

[19] datafactory AG, "World Wide Web," http://www.datafactory.de [last accessed 28.03.2005], 2005. 